Mental Health (Care and Treatment) (Scotland) Act 2003, does require some impairment of decision making - 'because of the mental disorder, the patient's ability to make decisions about the provision of medical treatment is significantly impaired' - but does not require that the person lacks the capacity to make the relevant decision in the way that is required for adults suffering from physical illnesses.

For patients in both jurisdictions, total control of the patient, including deprivation of liberty, is authorised if the criteria for detention are met. The authority is not limited to providing care and treatment solely in relation to the specific decision about which the patient is incapacitous.

The Irish Mental Health Act 2001 includes a similar requirement:

because of the severity of the illness, disability or dementia, the judgment of the person concerned is so impaired that failure to admit the person to an approved centre would be likely to lead to a serious deterioration in his or her condition or would prevent the administration of appropriate treatment that could be given only by such admission.

The Irish act also makes reference to 'best interests' but it is qualified:

In making a decision under this Act concerning the care or treatment of a person (including a decision to make an admission order in relation to a person), the best interests of the person shall be the principal consideration with due regard being given to the interests of other persons who may be at risk of serious harm if the decision is not made.

In New Zealand, the Mental Health (Compulsory Assessment and Treatment) Act 1992 requires that the disorder either causes a 'serious danger to the health or safety of that person or of others' or 'seriously diminishes the capacity of that person to take care of himself or herself'. This is similar to the law in Queensland, Australia (Mental Health Act 2000), which states that the person must either 'lack the capacity to consent to be treated for the illness' or 'has unreasonably refused proposed treatment for the illness'.

The mental health acts that make provision for compulsory treatment in the community tend to have a similar lack of regard for patient autonomy. A rare exception is from Canada, where the criteria for a community treatment order set out in Saskatchewan's Mental Health Services Act 2006 include the following:

The person is unable to understand and to make an informed decision regarding his or her need for treatment, care or supervision as a result of the mental disorder.

Ontario has a similar criterion: the person 'has shown or is showing a lack of competence to care for himself or herself' while living in the community,

It is not only nationally that the legal framework for the non-consensual care and treatment of people with a mental illness differs from the framework that applies to people who are physically ill. Article 5 of the European Convention on Human Rights (1950) is highly discriminatory. It groups together those of unsound mind, drug addicts, alcoholics and vagrants, as people who may legitimately be locked up, without any requirement that they have committed an offence or are incapable of making decisions for themselves, or even that they will personally benefit from the intervention.

The United Nations (UN) starts better with its Declaration of Human Rights (1948):

Recognition of the inherent dignity and of the equal and inalienable rights of all members of the human family is the foundation of freedom, justice and peace in the world.

Unfortunately, while it does better than most, it fails to maintain this high ideal. The UN Principles for the Protection of Persons with Mental Illness and the Improvement of Mental Health Care (UN General Assembly Resolution 46/119 of 17 December 1991) set out the grounds for compulsion:

O that, because of the mental illness, there is a serious likelihood of immediate or imminent harm to that person or to other persons; or

o that, in the case of a person whose mental illness is severe and whose judgement is impaired, failure to admit or retain that person is likely to lead to a serious deterioration in his or her condition or will prevent the giving of appropriate treatment that can be given only by admission to a mental health facility in accordance with the principle of the least restrictive alternative.

I return to where I started. Why? Why do people who are mentally ill need a different legal framework for their nonconsensual care and treatment?

\title{
Reproductive risk to maternal mental health: international perspectives
}

\section{David Skuse}

Behavioural and Brain Sciences Unit, Institute of Child Health, London WC1N 1EH, UK, email dskuse@ich.ucl.ac.uk

D espite the emphasis placed, in international comparisons of obstetric management, on the perinatal mortality rate as a measure of excellence of care (or otherwise), there has been relatively little discussion of the impact of birth on maternal mental health. When thinking about this issue, we need to consider both the mental state of the mother during the antenatal period as well as the subsequent impact of the birth on her mood and risk of major mental illness. According to the authors contributing to the theme discussed here, that risk is much higher 
than we might have predicted, in diverse communities in Pakistan, Nigeria and India. None of these communities has access to the professional support services that might ameliorate the consequences of reproductive risk.

Atif Rahman and Siham Sikander discuss work they have done in Pakistan to identify the prevalence of depression among women in selected rural districts, both before and after delivery. No less than a quarter had depression before the birth, and the great majority continued to be depressed soon afterwards. In follow-up studies, it appeared that in over half of these women the depression persisted for at least a year. As we might expect, the care given to their newborn children was suboptimal, contributing to poor infant growth and child morbidity. Important conclusions are reached about the nature of appropriate interventions and the implications for policy - highlighting the perennial problem that mental health is a low priority on the healthcare agenda in many parts of the world.

In the second of our themed articles, Abiodun Adewuya and Olutayo Aloba address the misconception that emotional distress after birth is rare in sub-Saharan Africa. Their work in Nigeria with pregnant women has shown that both depression and severe symptoms of anxiety are relatively common during the antenatal period, affecting up to a third of women. During the post-partum period, there is probably a similar proportion of Nigerian women with mild to moderate depressive symptoms to that found in other cultures around the world. As in Pakistan, mothers with persisting depression after childbirth are likely to have children who grow less well and who may be more susceptible to morbidity. In Nigeria, as in Pakistan, there is a social premium placed on male children and failure to produce a son is associated with social exclusion and marital disharmony.

Finally, Vikram Patel provides a fascinating insight into the association between gynaecological complaints and mental health among women in India. Remarkably, it appears that about half of all women interviewed in community surveys in that country complain of a gynaecological problem, most commonly an abnormal vaginal discharge. While it was assumed at one time that such discharges are related to sexually transmitted infections, evidence is emerging that throws doubt on that assumption. Why, then, do so many women in Indian rural communities complain of this symptom, which is often physiological and quite normal? The author presents data on the association between common mental disorders and the complaint of abnormal vaginal discharge, collected during a series of studies that attempted to clarify the natural history of the condition. The findings clearly indicate there is a complex relationship between worries and tensions in the everyday lives of women in rural India and their focus of concern on this symptom, which can be associated with diverse somatic symptoms. Vikram Patel makes recommendations for the integration of effective mental healthcare within emerging reproductive health programmes in India, with the objective of intervening in a vicious cycle that connects rising anxiety with an increased discharge.

\title{
Reproductive risk and its role in maternal mental health - perspectives from Pakistan
}

\author{
Atif Rahman ${ }^{1}$ and Siham Sikander ${ }^{2}$ \\ ${ }^{1}$ Professor of Child Psychiatry, University of Liverpool, Child Mental Health Unit, Alder Hey Children's Hospital NHS Foundation Trust, \\ Mulberry House, Eaton Road, Liverpool L12 2AP, UK, email Atif.Rahman@liverpool.ac.uk
}

${ }^{2}$ Human Development Research Foundation, Islamabad, Pakistan

\begin{abstract}
There is now an increased awareness of the high rates of depression among women with young children in impoverished communities. Poor maternal mental health affects the home environment, family life, child care and parenting. This paper summarises some of the issues related to the determinants, consequences and management of maternal mental health during and after pregnancy in Pakistan, a low-income country.
\end{abstract}

\section{Maternal mental health in pregnancy and after childbirth}

The mental health problem with the greatest public health implications is unipolar depression. Globally, clinical depression affects about $10-15 \%$ of women around the time of childbirth. Contrary to previous beliefs, high rates of depression, between two and three times greater than in industrialised countries, have been observed in women after childbirth in low-income countries (Rahman \& Prince, 2009).

We conducted a prospective study in rural Pakistan to determine the prevalence, outcome and risk factors associated with perinatal depression in a sample of 700 pregnant women (Rahman et al, 2003). The study was conducted in two rural sub-districts of Rawalpindi. The women were assessed for depression in the third trimester using culturally valid tools, and re-evaluated 3 months after they had given birth. Of 632 mothers assessed antenatally, 160 (25\%) met the ICD-10 criteria for a depressive episode. Of 541 mothers assessed postnatally, 151 (28\%) were diagnosed with ICD-10 depressive episode. Twenty-two mothers (4\%) developed a de novo depressive episode in the postnatal period, while 8 